\title{
Nonlinear Brownian motion - mean square displacement
}

\author{
W.Ebeling* \\ Institute of Physics, Humboldt University, \\ D-12489 Berlin, Germany
}

Receive April 5, 2004

The stochastic dynamics of self-propelled Brownian particles is studied by means of the Langevin and the Fokker-Planck approach. We model the driving by a nonlinear friction function which has a negative part at small velocities, leading to active Brownian motion of the particles. The mean square displacement is estimated analytically and compared with numerical simulations.

Key words: statistical mechanics, Brownian motion, nonlinear friction, selfpropelling, Fokker-Planck equations, mean square displacement

PACS: $05.40 .+j, 05.40 . J C$

\section{Introduction}

The standard theory of Brownian motion due to Einstein, Smoluchowski, Langevin, Fokker and Planck is based on the model at which a particle of mass $m$ moves in a dense medium which generates friction and random collisions [1]. The equation of motion is the Langevin equation and the corresponding equation for the probability is the Fokker-Planck equation. In order to explain the basic ideas of this essentially linear theory we start with the classical case of force-free Brownian particles under equilibrium conditions. The phenomenological method yields a Maxwellian distribution, which is in fact a "canonical distribution function"

$$
f_{0}(\mathbf{v})=C \exp \left[-\frac{m \mathbf{v}^{2}}{2 k_{\mathrm{B}} T}\right]
$$

The alternative method to derive this distribution starts from the Langevin equation

$$
\frac{\mathrm{d} \mathbf{v}}{\mathrm{d} t}=-\gamma_{0} \mathbf{v}+\left(2 D_{0}\right)^{1 / 2} \xi(t)
$$

*E-mail: ebeling@physik.hu-berlin.de 
$\left(\gamma_{0}\right.$ - friction coefficient, $D_{0}$ - diffusion constant). The corresponding Fokker-Planck equation reads

$$
\frac{\partial P(\mathbf{v}, t)}{\partial t}=\frac{\partial}{\partial \mathbf{v}}\left[\gamma_{0} \mathbf{v} P(v, t)+D_{0} \frac{\partial P(v, t)}{\partial \mathbf{v}}\right]
$$

This equation is solved using the Gaussian distribution

$$
f_{0}(\mathbf{v})=C \exp \left[-\frac{\gamma_{0} v^{2}}{2 D_{0}}\right]
$$

The Gaussian corresponds to the equilibrium Maxwell distribution just in the case that the so-called Einstein relation

$$
D_{0}=\frac{k_{\mathrm{B}} T \gamma_{0}}{m}
$$

holds. In this paper we will consider several generalizations of these classical results to nonlineaar friction which are in part based on the work of Klimontovich $[1,2]$. These generalizations refer to non-equilibrium cases, when the friction is not a constant, but a function of the velocities, and when equations (1) and (5) are not observed. In this case the system might be driven far away from equilibrium.

Let us replace $\gamma_{0}$ by a function $\gamma(v)$ and the constant $D_{0}$ also by a function $D(v)$. Then we speak about "nonlinear Brownian motion" $[1,2]$, while in other connection we speak about "active Brownian movement" [3-6]. The corresponding Langevin equation of motion reads:

$$
\frac{\mathrm{d} \mathbf{v}}{\mathrm{d} t}=\frac{1}{m} F(\mathbf{v})+\sqrt{2 D\left(v^{2}\right)} \xi(t) .
$$

Here $F(\mathbf{v})=-m \gamma\left(v^{2}\right) \mathbf{v}$ is the dissipative force acting on the particle and $D\left(v^{2}\right)$ is a diffusion function. The theoretical problems connected with these rather general equations have many interesting applications in different fields, such as in the theory of sound [7], in physical chemistry [8,9] and in biophysics [10].

A rather new application refers to the dynamics of charged grains in dusty plasmas. During the last decade the physics of charged grains has attracted much experimental and theoretical interest [11]. The dynamics of Coulomb clusters has been studied in various experiments [12-14]. Theoretical reviews discussing some of these phenomena can, e.g., be found in $[15,16]$. In several recent papers, the stochastic dynamics of grains have been analyzed in detail [18-22]. In particular, it could be shown that the Brownian motion of grains in a plasma exhibits many parallels to the theory of active Brownian particles [23]. Therefore we believe that the theory explained here may find applications not only to biological problems but also to the dynamics of grains in plasmas. 


\section{Velocity-dependent friction functions}

\section{Negative friction driven by energy input:}

Nonlinear friction forces driven by an external energy source was first used in the theory of sound developed by Helmholtz and Rayleigh. Rayleigh postulated for the friction function a quadratic expression:

$$
\gamma\left(v^{2}\right)=-\gamma_{1}+\gamma_{2} v^{2}=\gamma_{1}\left(\frac{v^{2}}{v_{0}^{2}}-1\right)
$$

with $\gamma_{1}<0$. This Rayleigh-Helmholtz-type model is a standard model studied in many papers on Brownian dynamics [2]. Klimontovich studied the solutions of the Fokker-Planck and Langevin equations for this model in much detail, including sustained oscillations, fluctuations, correlations etc. [2]. We note that $v_{0}^{2}=\gamma_{1} / \gamma_{2}$ defines a special value of the velocities where the friction changes from negative to positive values. This is the characteristic velocity of the problem, which defines e.g. the motion on limit cycles. Beside the Helmholtz-Rayleigh model we will study here a different model for active friction with energy input which was introduced and treated in $[3,4]$. This new friction function is based on a model of Brownian motion with energy depot. We assume that the Brownian particle itself should be capable of taking up external energy, thus storing some of this additional energy into an internal energy depot, $e(t)$. This energy depot may be altered by three different processes:

1. Take-up of energy from the environment with the rate $q$.

2. Internal dissipation, which is assumed to be proportional to the internal energy; the rate of energy loss is $c$.

3. Conversion of internal energy into motion, where $d$ is the rate of conversion of internal to kinetic degrees of freedom. This means that the depot energy may be used in order to accelerate motion with the acceleration dev.

The corresponding balance equation is

$$
\frac{\mathrm{d} e(t)}{\mathrm{d} t}=q-c e-\mathrm{d} e v^{2}
$$

and the equation of motion reads

$$
\frac{\mathrm{d} \mathbf{v}}{\mathrm{d} t}=-\gamma_{0} \mathbf{v}+\mathrm{d} e \mathbf{v}+\left(2 D_{0}\right)^{1 / 2} \xi(t)
$$

We assume that the acceleration is not subject to noise. This extension of the model is motivated by investigations of active biological motion, that relies on the supply of energy, which is dissipated by metabolic processes, but can be also converted into kinetic energy. Under adiabatic conditions $\mathrm{d} e / \mathrm{d} t=0$, the depot model leads to $e_{0}=q /\left(c+\mathrm{d} v^{2}\right)$ and thus to the effective friction function

$$
\gamma\left(v^{2}\right)=\gamma_{0}-\frac{q d}{c+\mathrm{d} v^{2}} .
$$


Due to the pumping, slow particles are accelerated and fast particles are damped. At certain conditions the active friction function has a zero

$$
v_{0}^{2}=\frac{q}{\gamma_{0}}-\frac{c}{d}
$$

corresponding to stationary velocities $v_{0}$, where the friction disappears. For 2-dimensional motion the deterministic trajectory of our system is then attracted by a cylinder in the $4 \mathrm{~d}$-space given by

$$
v_{1}^{2}+v_{2}^{2}=v_{0}^{2} .
$$

Here $v_{0}$ is the value of the stationary velocity which is for the Rayleigh-model given by $v_{0}^{2}=\gamma_{1} / \gamma_{2}$ and for the depot-model by $v_{0}^{2}=q_{0} / \gamma_{0}-c / d$.

\section{Negative friction driven by momentum exchange:}

Following $[21,23]$ let us consider a plasma with highly charged grains. Then the friction is determined at first by passive friction due to collisions of the grains with other particles and due to momentum exchange between grains and the plasma. Rather important are charging collisions due to ion absorption by grains. In this case both the friction coefficient $\gamma\left(v^{2}\right)$ and the related diffusion coefficient $D\left(v^{2}\right)$ in the Fokker-Planck equation (22) can be calculated explicitely [21,23]. Taking into account both contributions we find

$$
\begin{aligned}
\gamma\left(v^{2}\right) & =\gamma_{0}+\gamma_{\mathrm{i}}\left(v^{2}\right), \\
D\left(v^{2}\right) & =D_{0}+D_{\mathrm{i}}\left(v^{2}\right) .
\end{aligned}
$$

The explicit result for the friction function due to absorption effects, first derived in [21], reads

$$
\begin{aligned}
\gamma_{\mathrm{i}}\left(v^{2}\right) & =-3 A\left[I_{1}(\eta)+I_{2}(\eta, \Gamma)\right] \\
I_{1}(\eta) & =\left(-\frac{1}{2}+\frac{1}{4 \eta}\right) \sqrt{\frac{\pi}{\eta}} \operatorname{Erf}(\sqrt{\eta})-\frac{\mathrm{e}^{-\eta}}{2 \eta} \\
I_{2}(\eta) & =\frac{\Gamma}{\eta}\left[\frac{1}{2} \sqrt{\frac{\pi}{\eta}} \operatorname{Erf}(\sqrt{\eta})-\mathrm{e}^{-\eta}\right]
\end{aligned}
$$

with $\eta=\left(v^{2} / 2 v_{\mathrm{T}}^{2}\right)\left(v_{\mathrm{T}}\right.$-thermal velocity) and $\operatorname{Erf}(x)$ denoting the error function. The nonideality $\Gamma$ and the constant pre-factor $A$ depend on the grain and plasma parameters

$$
\Gamma=\frac{Z_{\mathrm{i}} Z_{\mathrm{g}} \mathrm{e}^{2}}{k_{\mathrm{B}} T a}, \quad A=\frac{1}{3} \sqrt{2 \pi}\left(\frac{m_{\mathrm{i}}}{m_{\mathrm{g}}}\right) a^{2} n_{\mathrm{i}} v_{\mathrm{T}},
$$

where $m_{g}$ is the grain mass, $a$ is the radius of the grain, $n_{\mathrm{i}}$ is the ion density in the plasma and $m_{\mathrm{i}}$ is the ion mass. The terms $I_{1}(\eta)$ and $I_{2}(\eta)$ are related to purely geometrical and charge-dependent collecting cross-sections, respectively. Correspondingly, it is also possible to calculate the related diffusion coefficient [21] The typical 


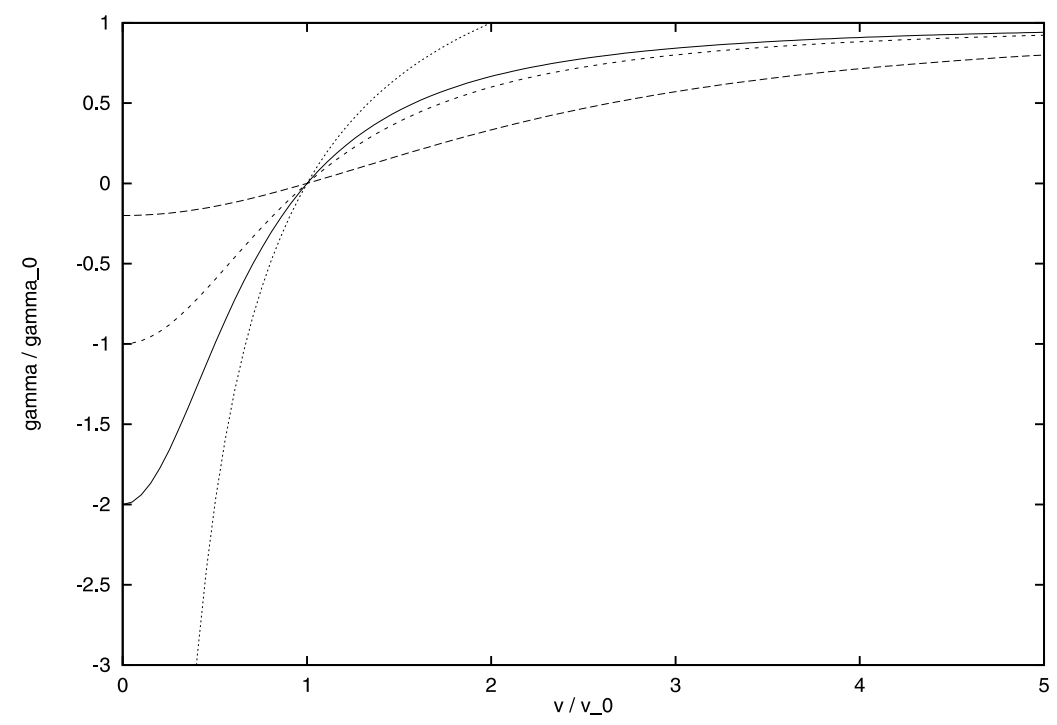

Figure 1. Friction function of the depot model for different parameters of driving.

behavior of $\gamma_{\mathrm{i}}(v)$ is rather similar to the friction function of the depot model shown in figure 1 . The function $D_{\mathrm{i}}(v)$ may be approximated by a constant.

\section{Approximations for the friction function:}

In many situations it is sufficient to approximate a more complicated friction coefficient $\gamma\left(v^{2}\right)$ by more simple models $[5,23]$. In the case of a rather weak driving force all our models may be unified, since in this case only the behaviour around $v^{2}=0$ is relevant. For the Rayleigh model, weak driving means $\gamma_{1} \ll \gamma_{0}$, and for the depot model it means

$$
\frac{q_{0}}{\gamma_{0}}>\frac{c}{d}, \quad \frac{q d}{c}-\gamma_{0} \ll 1 .
$$

Near to the bifurcation point where the friction changes to negative values, all models may be unified and by a Taylor expansion we obtain

$$
\gamma(v)=-\alpha+\beta v^{2} .
$$

The value of the constants is

$$
\alpha=-\gamma\left(v^{2}=0\right), \quad \beta=\gamma^{\prime}\left(v^{2}=0\right) .
$$

Far beyond the bifurcation point the system is strongly attracted to the two stable points $|\mathbf{v}|= \pm \mathbf{v}_{\mathbf{0}}$ where $v_{0}$ is the zero of the friction function. In this case the dissipative force may be well approximated by a piecewise linear approximation (Schienbein-Gruler model) which reads

$$
\mathbf{F}=-m \gamma_{1}\left[1-\frac{v_{0}}{|v|}\right] \mathbf{v} .
$$


The constant is determined by the derivative in the zero point:

$$
\gamma_{1}=2 \gamma^{\prime}\left(v_{0}^{2}\right) v_{0}^{2}
$$

This linear approximation to the friction force close to the two stable velocities $v= \pm v_{0}$ is very useful in many applications.

\section{Fokker-Planck equations and stationary solutions}

The Fokker-Planck equation (FPE) for the probability distribution function $f(\mathbf{r}, \mathbf{v})$ of the grain reads

$$
\frac{\partial f}{\partial t}+\mathbf{v} \frac{\partial f}{\partial \mathbf{r}}=\frac{\partial}{\partial \mathbf{v}}\left[\gamma\left(v^{2}\right) \mathbf{v} f+\frac{\partial D\left(v^{2}\right) f}{\partial \mathbf{v}}\right]
$$

A stationary solution of this equation is given by $[1,2,5,21,22]$

$$
f(\mathbf{v})=\frac{C}{D\left(v^{2}\right)} \exp \left(-\frac{1}{2} \int \frac{\gamma\left(v^{2}\right)}{D\left(v^{2}\right)} \mathrm{d} v^{2}\right)
$$

For a given friction function $\gamma\left(v^{2}\right)$ and a given diffusion function $D\left(v^{2}\right)$, the velocity distribution function can be readily obtained by integration. In case of a non-constant friction and diffusion functions, the stationary distribution will be non-Gaussian.

The friction and diffusion functions discussed above are rather complicated. The most essential point is the existing negative friction connected with a root of the friction function. Neglecting for a moment the diffusion effects the dynamical equation of a grain reads

$$
\frac{\mathrm{d}}{\mathrm{d} t} \mathbf{r}=\mathbf{v}, \quad \frac{\mathrm{d}}{\mathrm{d} t} \mathbf{v}=-\gamma\left(v^{2}\right) \mathbf{v}
$$

Multiplying by the velocity $\mathbf{v}$ we find for the kinetic energy of the grains

$$
\frac{\mathrm{d}}{\mathrm{d} t}\left(\frac{1}{2} \mathbf{v}^{2}\right)=-\gamma\left(v^{2}\right) \mathbf{v}^{2}
$$

Thus, we see that the kinetic energy of grains converges to the value $m_{\mathrm{g}} v_{0}^{2} / 2$; i.e., in the deterministic limit $D \rightarrow 0$ the root of the friction function determines the attractor of motion in the velocity space.

For dominant active friction, the dynamics of grains is quite different from that of ordinary thermal particles. Then a typical grain moves with constant absolute velocity but changes from time to time its direction due to the effect of noise. For the case of constant diffusion, $D\left(v^{2}\right) \equiv D_{0}$, we may easily write a Langevin equation

$$
\frac{\mathrm{d}}{\mathrm{d} t} \mathbf{v}+\gamma(v) \mathbf{v}=\sqrt{2 D_{0}} \xi(t)
$$




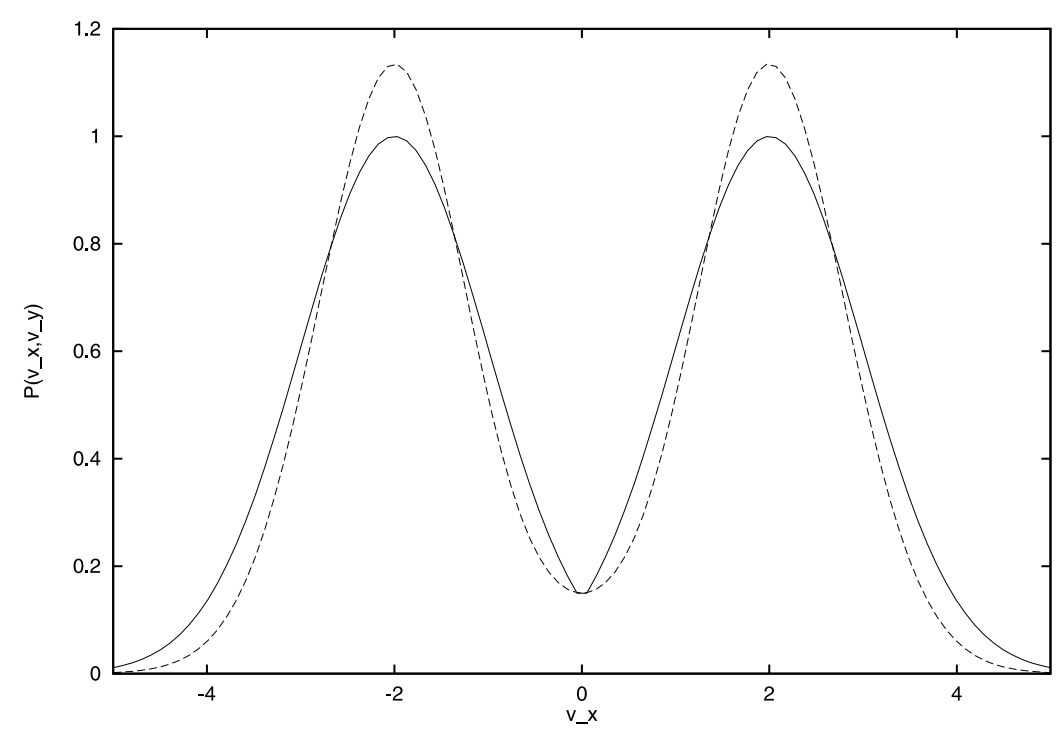

Figure 2. Stationary distribution function for the depot model and for the Schienbein-Gruler model.

where, as usual, $\xi(t)$ denotes a standard white noise source.

The solution of the corresponding Fokker-Planck equation (the velocity distribution function) reads for Rayleigh's model

$$
f(\mathbf{v})=\mathcal{N} \exp \left[-\frac{\gamma_{0}}{4 D_{0}}\left(v^{2}-v_{0}^{2}\right)^{2}\right]
$$

where $\mathcal{N}$ is a normalization constant. As one can see, the probability density $f(\mathbf{v})$ exhibits two peaks near $v_{0}$ and decays exponentially at high velocities. For the depot model the solution of equation (23) defining the stationary velocity distribution reads

$$
f(\mathbf{v})=\mathcal{N} \exp \left[-\frac{\gamma_{0}}{2 D_{0}} v^{2}+\frac{q}{2 D_{0}} \ln \left(1+\frac{d}{c} v^{2}\right)\right] .
$$

The mean square velocity is given by

$$
\left\langle\mathbf{v}^{2}\right\rangle=D_{0} \frac{\partial}{\partial \gamma_{0}} \mathcal{N}
$$

and the most probable velocity $\tilde{v}$ is the positive root of the bi-quadratic equation

$$
\frac{D_{0}}{\tilde{v}^{2}}+\frac{q d}{c+\mathrm{d} \tilde{v}^{2}}=\gamma_{0}
$$

The shape of the distribution functions is shown in figure 2. The depot model contains for $q \rightarrow 0$ the Maxwell distribution as a limiting case and yields for this limit

$$
\left\langle\mathbf{v}^{2}\right\rangle=2 \frac{k T}{m}, \quad \tilde{v}^{2}=\frac{k T}{m} .
$$


In the opposite case of large $q$ we find

$$
\left\langle\mathbf{v}^{2}\right\rangle=\tilde{v}^{2}=v_{0}^{2}
$$

For this case and also for weak noise, $\alpha / D_{0} \gg 1$, the stationary distribution functions become very narrow around $\pm v_{0}$. In the so-called $\delta$-limit, corresponding to $D_{0} / \alpha \rightarrow$ 0 , all distribution functions degenerate to

$$
f_{0}(\mathbf{v})=\tilde{\mathcal{N}} \delta\left(\mathbf{v}^{2}-\mathbf{v}_{\mathbf{0}}^{\mathbf{2}}\right),
$$

that is, the dispersion of the kinetic energy of the particles is completely neglected. From the point of view of physics, in this limit the particles move with constant absolute velocity, changing only their directions of motion from time to time.

\section{Mean square displacement}

For standard Brownian motion Einstein derived (in 1905), for the mean square displacement, the well-known relation

$$
\left\langle(\mathbf{r}(\mathbf{t})-\mathbf{r}(\mathbf{0}))^{2}\right\rangle=d \frac{2 k_{\mathrm{B}} T}{m \gamma_{0}} t
$$

where $d$ is the dimension. For the stochastic motion on the plane considered here we have $d=2$. For the case of non-linear Brownian motion it is much more difficult to obtain explicit solutions. Numerical experiments with the mean square displacement of the depot model were first carried out in [6]. Mikhailov and Meinköhn [9] were able to give analytical estimates for the mean square displacement in the limiting case of $\delta$-distributions of the velocities (negative friction and small noise) corresponding to equation (33). We will calculate here for the motion on the plane $d=2$ the mean square displacement for the depot model by generalizing the methods developed in $[1,2]$ and [9]. A particle starting at $t=0$ in $\mathbf{r}(\mathbf{0})$ will at time $t$ at the vector

$$
\mathbf{r}(t)=\int_{0}^{t} \mathrm{~d} t_{1} \mathbf{v}\left(t_{1}\right)
$$

The general expression for the mean square displacement reads [5]

$$
\left\langle\left(\mathbf{r}(t)-\mathbf{r}(0)^{2}\right\rangle=\int_{0}^{t} \mathrm{~d} t_{1} \int_{0}^{t} \mathrm{~d} t_{2}\left\langle\mathbf{v}\left(t_{1}\right) \mathbf{v}\left(t_{2}\right)\right\rangle\right.
$$

The correlation function of the velocities may be calculated exactly if the absolute velocities are constant and the change of directions is an uncorrelated random process [9]. We will make here a weaker assumption that the velocities have a rather narrow distribution around the most probable value $\tilde{v}$. Assuming that the velocities are close to the most probable value $\tilde{v}$

$$
\mathbf{v}(t)=\tilde{v} \mathbf{n},
$$


where $\mathbf{n}=\mathbf{v} /|\mathbf{v}|$ is the unit vector pointing in the direction $\mathbf{v}$. This way we get the mean square displacement

$$
\begin{aligned}
\left\langle\left(\mathbf{r}(t)-\mathbf{r}(0)^{2}\right\rangle\right. & =\tilde{v}^{2} K(t), \\
K(t)=\int_{0}^{t} \mathrm{~d} t_{1} \int_{0}^{t} \mathrm{~d} t_{2}\left\langle\mathbf{n}\left(\mathbf{t}_{1}\right) \mathbf{n}\left(\mathbf{t}_{\mathbf{2}}\right)\right\rangle & =\int_{0}^{t} \mathrm{~d} t_{1} \int_{0}^{t} \mathrm{~d} t_{2}\left\langle\cos \left(\phi\left(t_{1}-t_{2}\right)\right\rangle,\right.
\end{aligned}
$$

where $\phi$ is the angle between the unit vectors. Assuming that radial variable $\rho=|\mathbf{v}|$ is slowly changing, we find following Klimontovich [1] for the distribution of the angles

$$
\frac{\partial f(\phi, t)}{\partial t}=\frac{D_{0}}{2 \rho^{2}} \frac{\partial^{2}}{\partial \phi^{2}}
$$

We replace now $\rho^{2}$ by the mean value $\left\langle v^{2}\right\rangle$ and introduce the dimensionless time

$$
t_{0}=\frac{\left\langle v^{2}\right\rangle}{2 D_{0}}
$$

By using the known time-dependent distribution for the angles we get the correlation function

$$
K(t)=t_{0}^{2} k\left(t / t_{0}\right), \quad k(\tau)=[\exp (-\tau)-1+\tau] .
$$

This leads to the final expression

$$
\left\langle(\mathbf{r}(t)-\mathbf{r}(0))^{2}\right\rangle=4 \tilde{v}^{2} t_{0}^{2} k\left(t / t_{0}\right) .
$$

In the limit of passive Brownian motion $q=0$, this leads to

$$
\left\langle(\mathbf{r}(t)-\mathbf{r}(0))^{2}\right\rangle=\frac{4 k_{\mathrm{B}} T}{m \gamma_{0}^{2}}\left[t+\gamma_{0}^{-1}\left(\exp \left(-\gamma_{0} t\right)-1\right)\right] .
$$

We see that this limit is in full correspondence with the known results for passive Brownian motion, in particular for large time with equation (34) In the limit of strong driving ( $\delta$-limit we come back to the expression first derived by Mikhailov and Meinköhn [9]

$$
\left\langle(\mathbf{r}(t)-\mathbf{r}(0))^{2}\right\rangle=\frac{2 v_{0}^{4}}{D_{0}} t+\frac{v_{0}^{6}}{D_{0}^{2}}\left[\exp \left(-\frac{2 D_{0} t}{v_{0}^{2}}\right)-1\right] .
$$

This way we have shown that our approximate expression equation (40) is correct in the limits $q \rightarrow 0$ and $q \rightarrow \infty$. For intermediate values of the driving parameter $q$ our result equation (40) is at least a useful approximation. In order to check this we have carried out several simulations for the depot model. As we see in figures 3 and 4, the points found from numerical experiments are in reasonable agreement with the theoretical estimates. We underline that the spatial diffusion coefficient given here refers to the case of individual particles (self-diffusion). The expressions do not take into account interactions of the particles. For the case of cell movements the analytical expressions for the stationary velocity distribution and for the mean square displacement are in good agreement with measurements on granulocytes [10]. We also mention good agreement with earlier computer simulations [6]. 


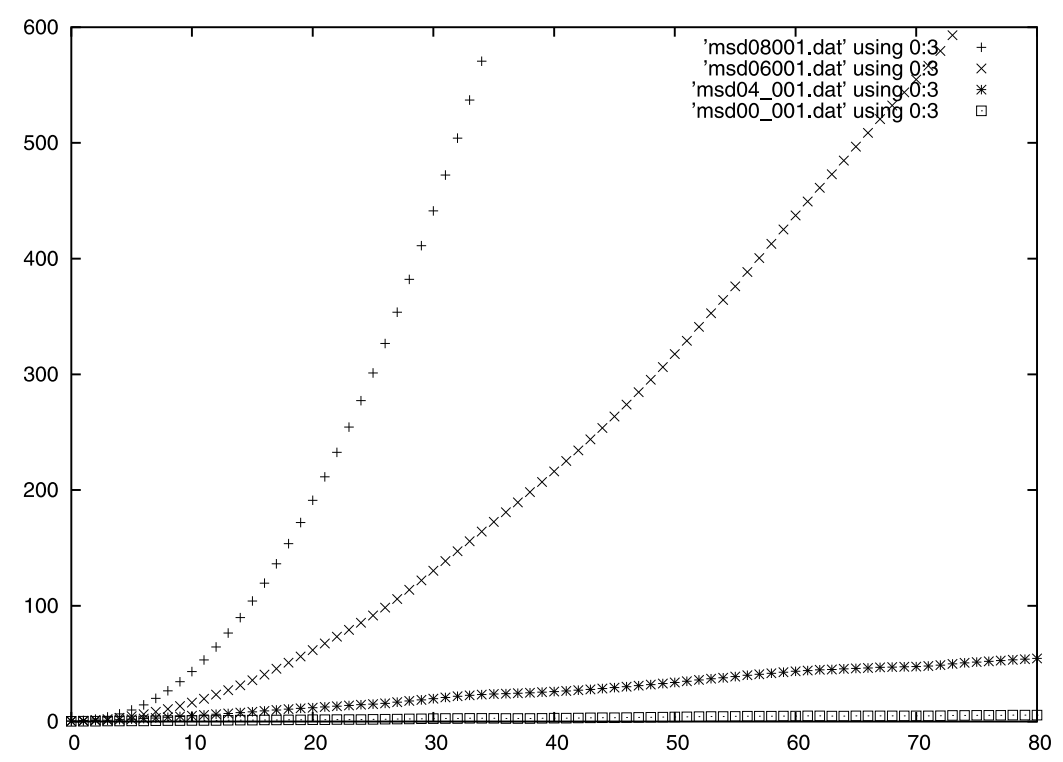

Figure 3. Mean square displacement as a function of time for the parameters $\gamma_{0}=0.5, c=d=1$ and several small values of the driving parameter $q$. The given curves give the mean square displacement for $q=0.0 ; 0.4 ; 0.6 ; 0.8$ as a function of time.

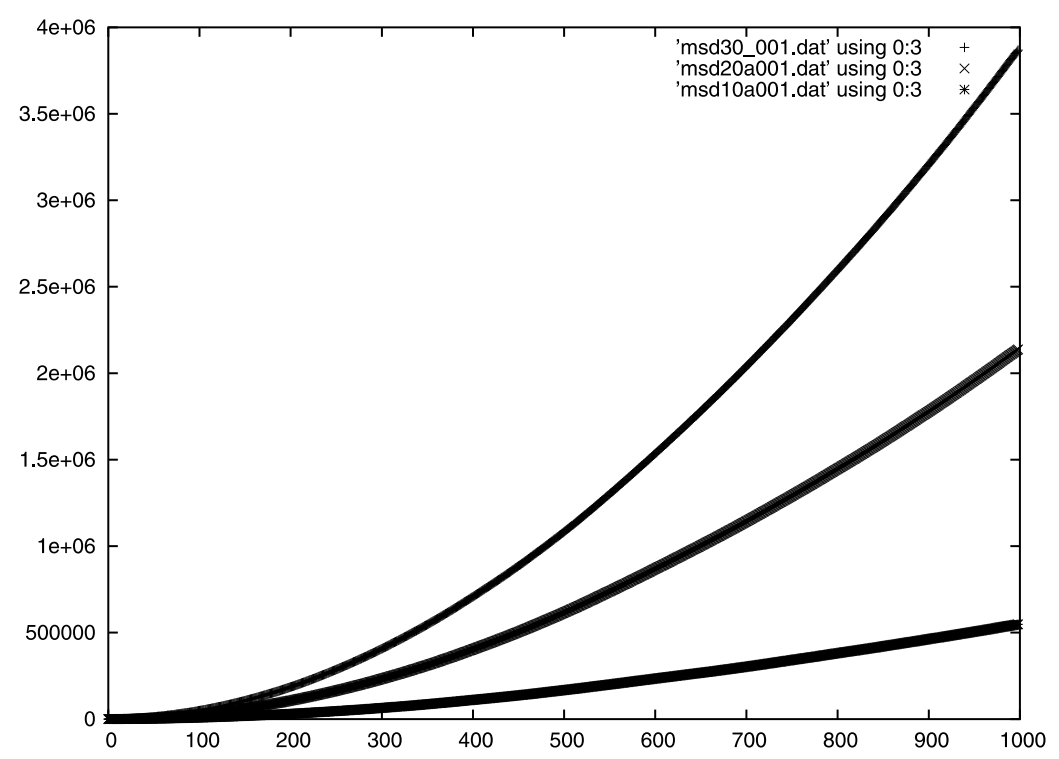

Figure 4. Mean square displacement as a function of time for the parameters $\gamma_{0}=0.5, c=d=1$ and several larger values of the driving parameter $q$. The given curves give the mean square displacement for $q=1.0 ; 2.0 ; 3.0$ as a function of time. 


\section{Discussion and conclusions}

The aim of this paper is to contribute new results to the theory of nonlinear Brownian motion. We investigated both the passive and the active case. While for passive Brownian motion the velocities are Gaussian-ditributed and centered around zero velocity, in the case of active motion the particles are driven to some characteristic modulus of the velocity $v_{0}$. Correspondingly all particles have nearly the same kinetic energy and differ only in directions. The mean square displacements are rather high. Following the methods developed in $[1,2,9]$ analytical estimates for the mean square displacement were derived, which show reasonable agreement with old and new simulations [6].

\section{Acknowledgements}

The author is grateful to J.Dunkel, L.Schimansky-Geier, S.Trigger and F.Schweitzer for helpful discussions.

\section{References}

1. Klimontovich Yu.L. Statistical physics. Moscow, Nauka, 1982, (in Russian); New York, Engl. transl. Harwood Academic Publ., 1986.

2. Klimontovich Yu.L. Statistical theory of open systems. Moscow, Janus, 1995, (in Russian); Amsterdam, Engl. transl. Kluwer Academic Publ., 1997.

3. Schweitzer F., Ebeling W., Tilch B. // Phys. Rev. Lett., 1998, vol. 80, p. 5044-5047.

4. Ebeling W., Schweitzer F., Tilch B. // BioSystems, vol. 49, p. 17-29.

5. Erdmann U., Ebeling W., Schimansky-Geier L., Schweitzer F. // Eur. Phys. J. B, 2000, vol. 15, p. 105.

6. Schweitzer F., Ebeling W., Tilch B. // Phys. Rev. E, 2001, vol. 64, p. 021110.

7. Rayleigh J.W. The Theory of Sound, vol. I, 2nd edition. New-York, Dover, 1945.

8. de Gennes P-G. // Rev. Mod. Phys., 1985, vol. 57, p. 827.

9. Mikhailov A.S., Meinköhn D. Stochastic Dynamics, (eds. Schimansky-Geier L., Pöschel T.), vol. 484. Lectures Notes in Physics. Berlin, Springer, 1997.

10. Schienbein M., H. Gruler // Bull. Math. Biology, 1993, vol. 55, p. 585.

11. Thomas H.M., Morfill. G.E. // Nature, 1996, vol. 379, p. 806.

12. Fortov V.E., Nefedov A.N., Petrov O.F. // Physics - Usphekhi, 1997, vol. 40, p. 1163.

13. Melzer A., Piel A., Klindworth M. // Phys. Rev. Lett., 2001, vol. 87, p. 115002.

14. Fortov V.E. et al. // Zh. exp. Theor. Fiz., 2003, vol. 123, p. 798.

15. Tsytovich V.N. // Physics - Uspekhi, 1997, vol. 40, p. 53.

16. Verheest F. Waves in Dusty Space Plasmas. Dordrecht, Kluwer, 2000.

17. Zagorodny A.G., Schram P.P.J.M., Trigger S.A. // Phys. Rev. Lett., 2000, vol. 84, p. 3594.

18. Schram P.P.J.M., Sitenko A.G., Trigger S.A., Zagorodny A.G. // Phys. Rev. E, 2000, vol. 63 , p. 016403 .

19. Trigger S.A. // Contrib. Plasma Phys., 2001, vol. 41, p. 331.

20. Ignatov A.M., Trigger S.A., Ebeling W., Schram P.P.J.M. // Phys. Lett., 2001, vol. A293, p. 141. 
21. Trigger S.A., Zagorodny A.G. // Contr. Plasma Phys., 2003, vol. 43, p. 381; http://arXiv.org/abs/physics/0211013, 2002.

22. Trigger S.A. // Phys. Rev. E, 2003, vol. 67, p. 046403.

23. Dunkel J., Ebeling W., Trigger S.A. Acrive and passive Brownian motion of charged particles. Preprint of the Institute of Physics, Humboldt University, 2004.

\title{
Нелінійний броунівський рух: середньоквадратичне зміщення
}

\author{
В.Ебелінг \\ Інститут фізики університету Гумбольдта, \\ D-12489 Берлін, Німеччина
}

Отримано 5 квітня 2004 р.

Досліджується стохастична динаміка саморухомих броунівських частинок в рамках формалізмів Ланжевена та Фоккер-Планка. Рушійна сила моделюється нелінійною функцією тертя, яка має від'ємні значення при малих швидкостях, що приводить до активного броунівського руху частинок. Середньоквадратичне зміщення частинок оцінюється аналітично та порівнюється з даними чисельних розрахунків.

Ключові слова: статистична механіка, броунівський рух, нелінійне тертя, саморухомі частинки, середньоквадратичне зміщення

PACS: $05.40 .+j, 05.40 . J C$ 